\title{
Consumers report lower confidence in their genetics knowledge following direct-to-consumer personal genomic testing
}

\author{
Deanna Alexis Carere, MS, CGC ${ }^{1,2}$, Peter Kraft, PhD ${ }^{1}$, Kimberly A. Kaphingst ${ }^{3,4}$, J. Scott Roberts, PhD ${ }^{5}$, \\ Robert C. Green, MD, MPH2,6,7; for the PGen Study Group
}

Purpose: The aim of this study was to measure changes to genetics knowledge and self-efficacy following personal genomic testing (PGT).

Methods: New customers of 23andMe and Pathway Genomics completed a series of online surveys. We measured genetics knowledge (nine true/false items) and genetics self-efficacy (five Likertscale items) before receipt of results and 6 months after results and used paired methods to evaluate change over time. Correlates of change (e.g., decision regret) were identified using linear regression.

Results: 998 PGT customers (59.9\% female; 85.8\% White; mean age $46.9 \pm 15.5$ years) were included in our analyses. Mean genetics knowledge score was $8.15 \pm 0.95$ (out of 9) at baseline and $8.25 \pm 0.92$ at 6 months $(P=0.0024)$. Mean self-efficacy score was $29.06 \pm 5.59$ (out of 35) at baseline and $27.7 \pm 5.46$ at 6 months $(P<0.0001)$; on each item, $30-45 \%$ of participants reported lower self-efficacy following PGT. Change in self-efficacy was positively associated with health-care provider consultation $(P=0.0042)$, impact of PGT on perceived control over one's health $(P<0.0001)$, and perceived value of PGT $(P<0.0001)$ and was negatively associated with decision regret $(P<0.0001)$.

Conclusion: Lowered genetics self-efficacy following PGT may reflect an appropriate reevaluation by consumers in response to receiving complex genetic information.

Genet Med advance online publication 26 March 2015

Key Words: direct-to-consumer genetic testing; genetics knowledge; literacy; personal genomic testing; self-efficacy
Health literacy has been defined as "the capacity to obtain, process, and understand basic health information and services needed to make appropriate health decisions." Inadequate health literacy is most common among elderly and minority populations and those with low socioeconomic status, ${ }^{2}$ and it has consistently ${ }^{3}$ been associated with increased hospitalization, ${ }^{4}$ less regular use of preventive medicine, ${ }^{5}$ reduced adherence to medical recommendations, ${ }^{6}$ and poorer health status. ${ }^{7}$ A subtype of health literacy is genetic literacy, which refers to "the capacity to obtain, process, understand, and use genomic information for health-related decision making." ${ }^{8}$ No systematic national assessments of genetic literacy have been performed; however, there is evidence to suggest that considerable confusion about genetics exists in the general population ${ }^{9}$ and that low genetic literacy is associated with low health literacy. ${ }^{10}$

The clinical genetics encounter provides an opportunity to promote genetic literacy, and studies have shown improvements in basic genetics knowledge ${ }^{11,12}$ and comprehension of genetic testing concepts, ${ }^{13}$ more accurate risk perception, ${ }^{14}$ and greater perceived personal control ${ }^{15}$ following clinical genetic counseling. Direct-to-consumer (DTC) personal genomic testing (PGT), through which individuals purchase commercial analysis and interpretation of a wide range of genetic variants, has been called a "novel milieu for health education," 16 with the potential to educate and empower consumers, increase health autonomy, and motivate self-guided education in genetics. ${ }^{17}$ Whether PGT actually affects consumer genetic literacy, however, remains unknown.

Among DTC PGT customers in the Impact of Personal Genomics (PGen) Study, we measured two components of genetic literacy: health-related genetics knowledge and perceived self-efficacy with genetics knowledge (defined as confidence in one's ability to use genetic information ${ }^{18}$ ). We sought to investigate two questions within this sample of customers: (i) Is there is a significant change in health-related genetics knowledge following PGT? (ii) Is there a significant change in confidence with health-related genetics knowledge following PGT?

${ }^{1}$ Program in Genetic Epidemiology and Statistical Genetics, Department of Epidemiology, Harvard School of Public Health, Boston, Massachusetts, USA; ${ }^{2}$ Division of Genetics, Department of Medicine, Brigham and Women's Hospital, Boston, Massachusetts, USA; ${ }^{3}$ Department of Communication, College of Humanities, The University of Utah, Salt Lake City, Utah, USA; ${ }^{4}$ Cancer Control and Population Sciences, Huntsman Cancer Institute, The University of Utah, Salt Lake City, Utah, USA; ${ }^{5}$ Department of Health Behavior and Health Education, University of Michigan School of Public Health, Ann Arbor, Michigan, USA; ${ }^{6}$ Harvard Medical School, Boston, Massachusetts, USA; ${ }^{7}$ Partners Personalized Medicine, Boston, Massachusetts, USA. Correspondence: Robert C. Green (rcgreen@genetics.med.harvard.edu) 


\section{MATERIALS AND METHODS}

\section{Study design and procedures}

The PGen Study was approved by the Partners Human Research Committee and the University of Michigan School of Public Health Institutional Review Board. Informed consent was obtained electronically from each participant before enrollment. Complete details of the study design and data collection procedures have been reported previously. ${ }^{19,20}$

New customers of 23 andMe ${ }^{21}$ and Pathway Genomics ${ }^{22}$ (Pathway) were recruited online after placing an order for DTC PGT between March and July 2012. Participants were invited to three web-based surveys administered by Survey Sciences Group (Ann Arbor, MI): the first at baseline, after testing was ordered but before receipt of results; the second $\sim 2$ weeks after results were viewed; and the third $\sim 6$ months after results were viewed. In total, 1,464 participants completed the baseline survey and were eligible for follow-up; of these, 1,046 (71.4\%) and 1,042 (71.2\%) submitted the 2-week and 6-month surveys, respectively. PGT results were returned to customers per standard company practice and then linked to survey data at the end of survey administration.

\section{Instruments}

At baseline we measured age, race/ethnicity, ${ }^{23}$ gender, income, education, PGT company, self-reported health (a single item from the 36-item Short Form Health Survey ${ }^{24}$ ), consultation with a health-care provider when deciding whether to order PGT (yes/no and type of health-care provider), prior use of PGT services (yes/no), current anxiety, health-related genetics knowledge (knowledge), and self-efficacy with health-related genetics knowledge (self-efficacy).

Current anxiety was measured with the two-item Generalized Anxiety Disorder scale. ${ }^{25}$ Frequency of each item (e.g., "Over the past 2 weeks, how often have you felt nervous, anxious, or on edge?") was answered on a four-category scale ( $0-3$ points), for a total possible score of 6 , and a score $\geq 3$ is considered a positive screen for Anxiety Disorder or Panic Disorder on the Generalized Anxiety Disorder scale.

Few validated measures of genetic literacy exist; moreover, those that do have been developed for use in specific groups (including undergraduate students ${ }^{26}$ and the general population of the late $1990 \mathrm{~s}^{27}$ ) or were designed to be administered verbally. ${ }^{28}$ Because none of these was deemed appropriate for online surveying of a highly educated, generally healthy population seeking commercial PGT in 2012, no preexisting, validated genetic literacy instruments were available for use in the PGen Study.

We therefore evaluated knowledge using nine true/false statements selected from existing measures of genetic literacy/knowledge $e^{26,27,29,30}$ to reflect the type of genetic information provided by PGT. A knowledge score was computed by summing the number of correct responses (maximum of 9). Self-efficacy was measured with a five-item scale based on one previously used by Kaphingst et al. ${ }^{31}$ in a study of PGT users and adapted from a scale first developed and used by Parrott et al. ${ }^{32}$ Participants rated their agreement with each item (e.g., "I am confident in my ability to understand information about genetics") on a seven-point Likert scale ranging from "strongly disagree" (1) to "strongly agree" (7). A self-efficacy score was computed by summing the ratings for each item (maximum of 35).

At the 6-month follow-up we asked whether the consumers had discussed their PGT results with a health-care provider (yes/no and type of health-care provider). We also measured decision regret related to PGT, current anxiety, the impact of PGT on perceived control over one's health, and the perceived financial value of PGT. Decision regret was measured with a validated, five-item scale. ${ }^{33}$ Agreement with each item (e.g., "The decision did me a lot of harm") was answered on a fivecategory Likert scale from "strongly disagree" (1) to "strongly agree" (5), and the mean score across items then was computed and converted to a total score out of 100. Current anxiety at the 6-month follow-up was measured with the two-item Generalized Anxiety Disorder scale, as described above. Single survey items were used to measure change in perceived control over one's health (e.g., "Having personal genomic testing made me feel like I have more control over my health") and perceived commercial value of PGT (e.g., "I feel that I got what I paid for"), with agreement measured on a five-point Likert scale from "strongly disagree" (1) to "strongly agree" (5).

\section{Statistical analyses}

Data for this analysis were obtained from PGen Study participants who submitted both baseline and 6-month surveys and for whom there were complete data for age, gender, race/ethnicity, education, knowledge, and self-efficacy. Descriptive statistics were computed to characterize baseline demographic characteristics of the study sample and to describe knowledge and self-efficacy performance. Cronbach $\alpha$ statistics were computed as a measure of internal consistency of the five self-efficacy scale items.

Multivariate linear regression models were used to evaluate associations between demographic characteristics and baseline knowledge and self-efficacy scores. In these and all further analyses, age was modeled as a continuous variable; Hispanic/ Latino ethnicity was modeled as a dichotomous variable; and race and education were modeled as four-category variables, as presented in Table 1.

McNemar exact tests were used to test the hypothesis that participants' performance would change, from baseline to 6-month follow-up, on each knowledge item. Similarly, paired $t$-tests were used on a per-item basis to test the hypothesis that participants' reported self-efficacy would change following PGT. Paired $t$-tests also were used to evaluate change in total knowledge and self-efficacy scores from baseline to the 6-month follow-up.

Because of the modest observed variability in knowledge over time, the remaining analyses were performed for self-efficacy only. We used multivariate linear regression models for change in self-efficacy score to evaluate, in turn, associations between 
Table 1 Baseline demographics $(n=998)$

\begin{tabular}{|c|c|c|}
\hline & No. & $\%$ \\
\hline Male & 400 & 40.1 \\
\hline \multicolumn{3}{|l|}{ Race } \\
\hline White & 856 & 85.8 \\
\hline African-American & 23 & 2.3 \\
\hline Asian & 32 & 3.2 \\
\hline More than one race/other & 87 & 8.7 \\
\hline Hispanic/Latino ethnicity & 50 & 5.0 \\
\hline \multicolumn{3}{|l|}{ Education } \\
\hline Less than a College degree & 203 & 20.3 \\
\hline College degree & 304 & 30.5 \\
\hline Some graduate school & 359 & 36.0 \\
\hline Doctoral-level degree & 132 & 13.2 \\
\hline \multicolumn{3}{|l|}{ Income $(\$)$} \\
\hline$<100,000$ & 559 & 56.0 \\
\hline $100,000-199,999$ & 302 & 30.3 \\
\hline$\geq 200,000$ & 126 & 12.6 \\
\hline Unknown & 11 & 1.1 \\
\hline \multicolumn{3}{|l|}{ Self-reported health } \\
\hline Excellent & 149 & 14.9 \\
\hline Very good & 401 & 40.2 \\
\hline Good & 295 & 29.6 \\
\hline Fair & 110 & 11.0 \\
\hline Poor & 41 & 4.1 \\
\hline Unknown & 2 & 0.2 \\
\hline Positive GAD-2 screen for panic/anxiety disorder ${ }^{a}$ & 158 & 15.8 \\
\hline \multicolumn{3}{|l|}{ Health care provider consultation before PGT } \\
\hline Genetics specialist & 5 & 0.5 \\
\hline Other health care provider & 15 & 1.5 \\
\hline \multicolumn{3}{|l|}{ PGT company } \\
\hline 23andMe & 616 & 61.7 \\
\hline Pathway & 382 & 38.3 \\
\hline Prior PGT (different company) & 103 & 10.3 \\
\hline \multicolumn{3}{|l|}{ Age, years } \\
\hline Mean (range) & \multicolumn{2}{|c|}{$46.8(19-94)$} \\
\hline Standard deviation & \multicolumn{2}{|c|}{15.5} \\
\hline
\end{tabular}

GAD-2, two-item Generalized Anxiety Disorder screener; PGT, personal genomic testing.

The GAD-2 instrument provides a score between 0 and 6 . A score $\geq 3$ suggests a panic or anxiety disorder.

change in self-efficacy score and each of health-care provider consultation after PGT, decision regret, anxiety at the 6-month follow-up, reported change in perceived control over health, and perceived value of PGT. All models were adjusted for baseline self-efficacy score, age, gender, race/ethnicity, education, and PGT company; the model for the association between self-efficacy score and anxiety at the 6-month follow-up was additionally adjusted for the score on the two-item Generalized Anxiety Disorder tool at baseline.

Because health-care provider consultation after PGT measured an action temporally placed between baseline self-efficacy and 6-month self-efficacy, and because of a particular interest in the role of health-care providers in DTC PGT, we further examined the association between health-care provider consultation and change in performance on each self-efficacy item using multivariate linear regression.

All analyses were conducted using SAS software (version 9.3; SAS Institute, Cary, NC), and linear regression models were fitted using PROC GLM. Statistical significance for all analyses was set at $P<0.05$.

\section{RESULTS}

A total of 1,042 PGen Study participants submitted baseline and 6-month follow-up surveys, of which 44 were excluded from analysis because of missing knowledge or self-efficacy data at the 6-month follow-up. Demographic characteristics of the 998 participants included in our analyses are presented in Table 1.

\section{Genetics knowledge}

At baseline, knowledge scores ranged from 4 (44\% correct) to $9(100 \%$ correct), with a mean score of 8.15 (standard deviation $=0.95$ ). In a multivariate model for baseline knowledge score, including age, gender, education, race/ethnicity, and PGT company, male gender $(\beta=0.13, P=0.03)$ and higher levels of education $\left(\beta_{\text {college }}=0.31, P=.0003 ; \beta_{\text {graduate }}=0.32, P<0.0001\right.$; $\beta_{\text {doctorate }}=0.57, P<0.0001 ; P<0.0001$, global $F$-test $)$ were associated with higher baseline scores, whereas Hispanic/Latino ethnicity $(\beta=-0.69, P<0.0001)$ and older age $(\beta=-0.008$ per year, $P<0.0001)$ were associated with lower baseline scores.

At the 6-month follow-up, scores again ranged from 4 to 9 , but the mean knowledge score showed a significant increase of 0.10 units to 8.25 (standard deviation $=0.92 ; P=0.0024$, paired $t$-test). Approximately half of the participants $(n=509,51 \%)$ showed no change in knowledge score, whereas 191 participants $(19.1 \%)$ improved by 1 point and 81 participants $(8.1 \%)$ improved by 2 or more points. Most participants $(79.6 \%$ at baseline; $83.6 \%$ at the 6 -month follow-up) received a score $\geq 8$ at both time points, and a plurality received perfect scores at both time points (44.2\% at baseline; $49.0 \%$ at the 6 -month follow-up).

Item-specific performance over time is presented in Table 2. Performance was poorest on item 4 ("Most genetic disorders are caused by only a single gene"), with 63.8 and $68.1 \%$ of participants answering correctly at baseline and the 6-month follow-up, respectively. The proportion of correct responses surpassed $85 \%$ on all other items at both time points. On a per-item basis, a significant improvement in performance was observed only for items $4(P=0.0134$, paired $t$-test) and 8 ("A healthy lifestyle can prevent or lessen the negative consequences of having genetic predispositions to some disease"; $95.5 \%$ were correct at baseline versus $97.9 \%$ correct at the 6-month follow-up; $P=0.0022$ ).

\section{Genetics self-efficacy}

At baseline, self-efficacy scores ranged from 5 ("strongly disagree" with all five statements) to 35 ("strongly agree" with all five statements), with a mean score of 29.06 (standard deviation $=5.59$ ). In a multivariate model for baseline self-efficacy score, 
including age, gender, education, race/ethnicity, and PGT company, only education was positively associated with baseline score $\left(\beta_{\text {college }}=0.34, P=0.50 ; \beta_{\text {graduate }}=1.01, P=0.0404\right.$; $\beta_{\text {doctorate }}=2.49, P<0.0001 ; P=0.0004$, global $F$-test $)$.

At the 6-month follow-up, scores again ranged from 5 to 35 , but mean self-efficacy score showed a significant decrease of 1.35 units to 27.71 (standard deviation $=5.46 ; P<0.0001$, paired $t$-test). Approximately one-fifth of the participants ( $n=189,18.9 \%)$ showed no change in self-efficacy score, whereas 385 participants (38.6\%) indicated a decrease of one to five points, and $153(15.3 \%)$ indicated a decrease of more than five points. At baseline, $43.7 \%$ of participants agreed or strongly agreed with all five self-efficacy statements, whereas 6 months following PGT, 34.7\% of participants did so. Cronbach $\alpha$ values for the five self-efficacy items at baseline and the 6-month follow-up were 0.94 and 0.95 , respectively, suggesting excellent internal consistency across items.

Item-specific performance over time is presented in Table 3. The proportion of participants reporting that they agreed or strongly agreed with each item varied by item and survey time point; items $1,2,3$, and 5 showed significant decreases $(P<0.0001)$ of $9.7-18.0$ percentage points from baseline to the 6-month follow-up. There was a small, nonsignificant decrease in agreement with item $4(64.3 \%$ at baseline versus $61.7 \%$ at the 6-month follow-up; $P=0.1536$ ). On each item, $30-50 \%$ of participants reported lower self-efficacy after PGT compared with baseline.

\section{Correlates of change in genetics self-efficacy following PGT}

Six months after receiving their PGT results, 348 participants (34.9\%) had shared their results with a health-care provider; of these, $272(27.3 \%)$ had shared with a primary care provider, $30(3.0 \%)$ with a genetics specialist (e.g., genetic counselor, medical geneticist), and 159 (15.9\%) with some other medical specialist. In a multivariate model, health-care provider consultation was positively associated with change in self-efficacy score from baseline to 6-month follow-up (Table 4): among

Table 2 Longitudinal performance on a measure of genetics knowledge among partiripants in the PGen study

\begin{tabular}{|c|c|c|c|c|}
\hline Genetics knowledge & \multicolumn{2}{|c|}{ Correct response, $\boldsymbol{n}(\%)$} & $\begin{array}{l}\text { Response at } 6 \text { months } \\
\text { changed to incorrect/ } \\
\text { to correct, } n(\%)\end{array}$ & $P$ value* \\
\hline 1. Healthy parents can have a child with an inherited disease (True) & $990(99.2)$ & $990(99.2)$ & $8(0.8) / 8(0.8)$ & 1.00 \\
\hline $\begin{array}{l}\text { 3. Some genetic disorders occur more often within particular ethnic } \\
\text { groups (True) }\end{array}$ & $990(99.2)$ & $992(99.4)$ & $5(0.5) / 7(0.7)$ & 0.77 \\
\hline $\begin{array}{l}\text { 6. A disease is only genetically determined if more than one family } \\
\text { member is affected (False) }\end{array}$ & $877(87.9)$ & $878(88.0)$ & $78(7.8) / 79(7.9)$ & 1.00 \\
\hline 7. Some genetic disorders occur later in adult life (True) & $930(93.2)$ & $948(95.0)$ & $40(4.0) / 58(5.8)$ & 0.09 \\
\hline $\begin{array}{l}\text { 8. A healthy lifestyle can prevent or lessen the negative consequences } \\
\text { of having genetic predispositions to some diseases (True) }\end{array}$ & $953(95.5)$ & $977(97.9)$ & $17(1.7) / 41(4.1)$ & 0.0022 \\
\hline
\end{tabular}

*P value obtained from McNemar exact tests

Table 3 Longitudinal self-efficacy with health-related genetics concepts among participants in the PGen study

\begin{tabular}{|c|c|c|c|c|c|c|c|}
\hline \multirow[b]{2}{*}{ Genetic self-efficacy } & \multicolumn{3}{|c|}{ Rating, a mean (SD) } & \multicolumn{3}{|c|}{ Agree or strongly agree (\%) } & \multirow{2}{*}{$\begin{array}{c}\text { Changed response at } \\
6 \mathrm{M}, n(\%) \text { increase/ } \\
\text { decrease }\end{array}$} \\
\hline & BL & $6 \mathrm{M}$ & $\begin{array}{c}P \\
\text { value* }^{*}\end{array}$ & BL & $6 \mathrm{M}$ & $\begin{array}{c}P \\
\text { value** }\end{array}$ & \\
\hline $\begin{array}{l}\text { 1. I am confident in my ability to understand } \\
\text { information about genetics. (Genetics) }\end{array}$ & $6.06(1.18)$ & $5.60(1.25)$ & $<0.0001$ & 79.5 & 62.2 & $<.0001$ & $126(12.6) / 457(45.8)$ \\
\hline $\begin{array}{l}\text { 2. I am able to understand information about } \\
\text { how genes can affect my health. (Health) }\end{array}$ & $6.15(1.09)$ & $5.72(1.12)$ & $<0.0001$ & 82.7 & 64.7 & $<.0001$ & $118(11.8) / 448(44.9)$ \\
\hline $\begin{array}{l}\text { 3. I have a good idea about how genetics may } \\
\text { influence risk for disease generally. (Disease) }\end{array}$ & $5.91(1.19)$ & $5.75(1.10)$ & $<0.0001$ & 73.6 & 63.9 & $<.0001$ & $180(18.0) / 343(34.4)$ \\
\hline $\begin{array}{l}\text { 5. I am able to explain to others how genes } \\
\text { affect one's health. (Explain) }\end{array}$ & $5.31(1.45)$ & $5.01(1.45)$ & $<0.0001$ & 49.8 & 38.7 & $<.0001$ & $202(20.2) / 409$ (41.0) \\
\hline
\end{tabular}

6M, 6-month follow-up; BL, baseline; SD, standard deviation.

a Likert rating scale from 1 (strongly disagree) to 7 (strongly agree). *Pvalue obtained from paired $t$-tests. ${ }^{*} P$ value obtained from McNemar exact tests. 
Table 4 Correlates of change in total genetic self-efficacy (GSE) score

\begin{tabular}{|c|c|c|c|}
\hline & Frequency, $n(\%)$ & $B_{\text {adjusted }}{ }^{a}$ & $P$ value \\
\hline Consultation with health care provider after PGT & $348(34.9)$ & $0.96 \pm 0.33$ & 0.0042 \\
\hline Positive GAD-2 screen for anxiety/panic disorder at 6-month follow-up & $145(14.5)$ & $-0.68 \pm 0.48^{b}$ & 0.1580 \\
\hline "Having personal genomic testing made me feel like I have more control over my health." & - & - & $<0.0001 *$ \\
\hline Strongly disagree & $64(6.4)$ & Reference & - \\
\hline Somewhat disagree & $74(7.4)$ & $0.06 \pm 0.82$ & 0.9423 \\
\hline Neither agree nor disagree & $197(19.7)$ & $1.88 \pm 0.69$ & 0.0064 \\
\hline Somewhat agree & $448(44.9)$ & $2.22 \pm 0.64$ & 0.0005 \\
\hline Strongly agree & $215(21.6)$ & $3.61 \pm 0.68$ & $<0.0001$ \\
\hline "I feel that I got what I paid for." & - & - & $<0.0001$ * \\
\hline Strongly disagree & $18(1.8)$ & Reference & - \\
\hline Somewhat disagree & $31(3.1)$ & $1.37 \pm 1.41$ & 0.3313 \\
\hline Neither agree nor disagree & $112(11.2)$ & $3.90 \pm 1.21$ & 0.0013 \\
\hline Somewhat agree & $308(30.9)$ & $4.66 \pm 1.15$ & $<0.0001$ \\
\hline Strongly agree & $529(53.0)$ & $5.97 \pm 1.14$ & $<0.0001$ \\
\hline Decision regret score, mean \pm SD $($ range $=0-100)$ & $7.59 \pm 13.7$ & $-0.09 \pm 0.44$ & $<0.0001$ \\
\hline
\end{tabular}

GAD-2, two-item Generalized Anxiety Disorder screener; PGT, personal genomic testing; SD, standard deviation.

${ }^{a}$ All models adjusted for baseline self-efficacy score, age, gender, race, ethnicity, education, and PGT company. ${ }^{b}$ Additionally adjusted for result of baseline GAD-2 screen for anxiety/panic disorder.

*P value from global $F$-test for the categorical variable.

participants who did not share their results with a health-care provider, the least squares-adjusted mean change in self-efficacy score was -1.88 (standard deviation $=0.38$ ) compared with a mean change of -0.93 (standard deviation $=0.44$ ) among those who did share their results with a health-care provider $\left(P_{\text {difference }}=0.0042\right)$. Health-care provider consultation also was significantly associated with change on each selfefficacy item, with the exception of item 3 ("I have a good idea about how genetics may influence risk for disease generally"), although this item showed a similar trend (Figure 1).

There was no significant difference in the proportion of participants with a positive screen for anxiety at the 6-month follow-up (14.5\%) compared with baseline (15.8\%; $P$ value $=0.33$, McNemar exact test ) and no significant association between a positive screen for anxiety at the 6-month followup and change in self-efficacy score. After adjusting for baseline self-efficacy score, age, gender, race/ethnicity, education, and PGT company, an increase in perceived control of one's health $(P<0.0001)$ and perceived financial value of PGT $(P<0.0001)$ each were positively associated with a change in self-efficacy score following PGT, whereas greater decision regret was negatively associated with change in self-efficacy score following PGT $(P<0.0001)$ (Table 4$)$. Decision regret following PGT was, however, quite rare: 583 participants (58.4\%) received a score of $0 / 100$ (no decision regret), and $972(97.4 \%)$ received a score of $40 / 100$ or less.

\section{DISCUSSION}

DCT PGT customers who enrolled in the PGen Study demonstrated high levels of genetics knowledge both before and after testing. Consistent with prior studies of health literacy ${ }^{2}$ and genetic literacy, ${ }^{10}$ genetics knowledge was positively associated

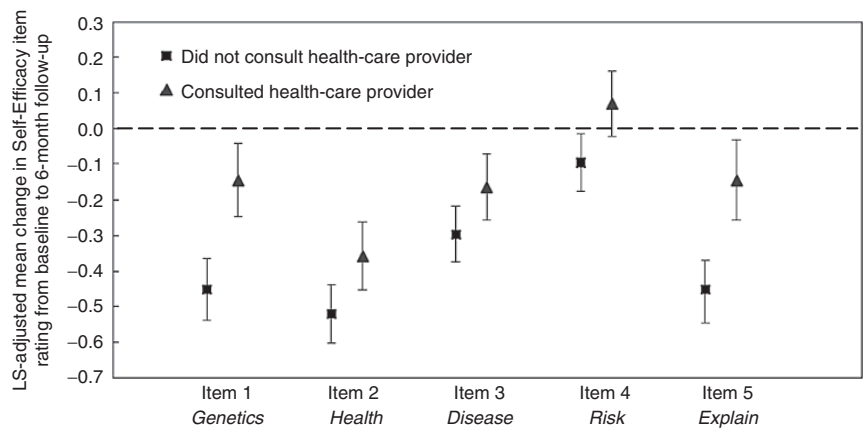

Figure 1 Least squares-adjusted mean change in rating of each selfefficacy item from baseline to the 6-month follow-up, stratified by health-care provider consultation status after personal genomic testing (PGT). Adjusted means were obtained from linear regression models for change in rating of each item, with adjustment for baseline item rating, age, gender, race/ethnicity, education, and PGT company. Health-care provider consultation was significantly associated with change in rating of items $1(P=0.0041), 2(P=0.0253), 4(P=0.0208)$, and $5(P=0.0003)$ but not item $3(P=0.0590)$.

with higher levels of education, younger age, and non-Hispanic/Latino ethnicity. In contrast to prior studies of both health literacy ${ }^{2}$ and genetic literacy, ${ }^{29}$ in which female gender was associated with higher levels of literacy, we found here a significant association between male gender and higher genetics knowledge. The reason for this discrepancy is not immediately obvious; however, it should be noted that male gender was associated with only a small increase in performance $(0.13$ points on a 9-point scale). Moreover, genetics knowledge was universally high in the PGen Study cohort (particularly when compared with the general population ${ }^{9}$ ), suggesting that individuals with high levels of genetics knowledge are more likely to seek out PGT services. 
Unlike genetics knowledge, greater genetics self-efficacy was associated with only education level and not with other baseline demographic characteristics. Because this is the first study to identify predictors of genetics self-efficacy, this finding should be followed up with further investigation in the general population and other populations undergoing genetic testing.

A statistically significant but small increase in genetics knowledge ( 0.10 points out of 9 ) was observed at the 6 -month followup; however, a ceiling effect was expected given strong baseline performance. These results provide modest evidence for an educational effect of the PGT experience; they also highlight the need for more sensitive measures of genetics knowledge that can be used among highly educated and informed users of new technologies to both evaluate static genetics knowledge and detect subtle changes to understanding over time.

Performance was poorest at both time points on item 4 ("Most genetic disorders are caused by only a single gene"), with fewer than $70 \%$ of participants responding correctly. This particular misconception is notable because the PGT provided to these customers largely focused on complex, non-Mendelian traits attributable to multiple genetic variants and nongenetic factors. That improvement on this item was so minimal (and included 123 participants who correctly answered "false" at baseline changed their response to "true" at the 6-month follow-up) suggests that, even after receiving a personalized genetic risk assessment, customers may still lack a sophisticated understanding of the genetic etiology of complex disease.

We observed a significant decrease from baseline to 6-month follow-up in overall genetics self-efficacy and in item-specific ratings for items $1,2,3$, and 5 . On these items, $34-46 \%$ of participants reported lower genetics self-efficacy at the 6-month follow-up, whereas only 11-20\% reported higher genetics selfefficacy after PGT. Notably, performance on item 4 ("I have a good idea about how my own genetic makeup might affect my risk for disease")-the item most directly related to the PGT experience-did not significantly decrease following PGT. On the other hand, there was no significant increase in performance on item 4 either, with slightly more participants reporting a negative change in confidence (32.1\%) than a positive change $(28.1 \%)$ at the 6-month follow-up.

One interpretation of our findings is that PGT customers, before receiving their PGT results, overestimated their grasp of complex disease genetics and thus had inflated perceptions of self-efficacy. Through the process of PGT-including the provision of dozens of results detailing both environmental and genetic contributions to disease and lengthy reports highlighting the inherent limitations of genetic risk assessment-participants improved their genetics knowledge and perhaps became aware of previously unrecognized complexities of genetics, thus becoming less confident in their understanding. Arguably, this is an appropriate and even expected response to the experience of PGT among nonexpert individuals with high baseline levels of genetics self-efficacy engaging with a novel genomic technology.
Other interpretations of our findings are also possible. For example, PGT may have inappropriately reduced participants' genetics self-efficacy. Perceived self-efficacy predicts an individual's ability to perform a particular action; however, self-efficacy is also shaped by attempts to perform that action. ${ }^{34}$ If PGT consumers were to perceive a challenge to their attempts to learn about their genetic risk of disease (for example, the use of technical jargon in results reports ${ }^{35}$ ), then genetics self-efficacy could be negatively affected by the PGT experience.

Regardless of the mechanism underlying the decrease in genetics self-efficacy, our results hint at a means of supporting and promoting consumer self-efficacy: Both before and after PGT, participants were least confident in their ability to understand how their own genetic makeup affects their risk for disease (item 4) and to explain to others how genes affect one's health (item 5). Notably, risk assessment/counseling and facilitation of family sharing fall within the scope of practice of certified genetic counselors ${ }^{36}$ and are integral to the clinical genetic counseling encounter, ${ }^{37}$ whether performed by a certified genetic counselor, medical geneticist, or other health professional. These results, together with the finding that consultation with a health-care provider after PGT was positively associated with both overall and item-specific change in genetics self-efficacy, suggest that greater engagement of health-care providers (either ones made available by the companies or customers' own providers) in the testing process may have the potential to positively impact genetics self-efficacy.

Given the small number of participants who reported consulting a genetics specialist or other medical specialist, we were unable to evaluate how changes to self-efficacy might differ depending on the type of health-care provider consulted. In the future, should the use of PGT and its incorporation into medical care become more common, studies that compare a range of service delivery models (e.g., genetic counselor-mediated PGT, primary care provider-mediated PGT, and pure DTC PGT) could help elucidate the nature of the relationship between consultation and consumer self-efficacy. In the meantime, and in light of our findings here, we suggest that all current and future studies of PGT users would benefit from consistent evaluation of longitudinal genetics self-efficacy, in addition to the more commonly measured outcome of genetics knowledge, to permit the comparison of longitudinal trends in self-efficacy across different cohorts.

Even after the observed decrease in genetics self-efficacy following PGT, genetics self-efficacy levels were still moderately high, with mean scores ranging from 5.01 to 5.75 ("somewhat agree") out of 7 at the 6-month follow-up. Nonetheless, we noted significant associations between change in genetics selfefficacy and certain measures of the PGT customer experience, including decision regret, perceived financial value of PGT, and reported impact of PGT on perceived control over one's health. Although our study design does not permit investigation of the causal relationship between change in genetics self-efficacy and each of these correlates, we suggest it is at least plausible that interventions to improve genetics self-efficacy could also 
reduce decision regret and increase perceptions of value and control among PGT customers.

Critics of DTC PGT have suggested that without mediation through a health-care provider, exposure to genetic risk information could lead to needless worry and increase the risk for clinical anxiety in customers. ${ }^{38}$ One mechanism for increased anxiety is through a reduction in self-efficacy, for example, when anxiety is aroused in an individual who perceives himor herself to be ill-equipped to handle a particular challenge. ${ }^{39}$ Here, however, we observed no significant difference in the proportion of customers with a positive screen for anxiety disorder following PGT and found no association between anxiety at the 6-month follow-up and change in self-efficacy score.

Strengths of the PGen Study include its large sample size, recruitment of actual PGT customers, and longitudinal data collection. This is the first study to measure both genetics knowledge and self-efficacy among PGT users, and to do so longitudinally, thus providing a dynamic picture of customer knowledge and confidence over the course of PGT.

Limitations of this study include the potential for selection bias inherent to voluntary survey data and the use of nonvalidated scales for genetics knowledge and self-efficacy. We also are unable to delineate the causal relationship between change in genetics self-efficacy and its correlates, such as health-care provider consultation; that is, it may be the case that highly selfefficacious consumers are more likely to engage in discussions of their results with a health-care provider, not that health-care provider consultation has a positive impact on genetics selfefficacy. Finally, our findings are generalizable only to consumers obtaining DTC PGT, and PGen Study participants tended to be well educated, high earning, and White; thus, the impact of PGT on genetics knowledge and self-efficacy may differ in groups without these qualities, particularly among those with low baseline health literacy or self-efficacy.

In conclusion, PGT may serve as an educational intervention in genetics for its consumers, but more sensitive measures of genetics knowledge are needed to answer this question among highly educated and informed populations, including PGT customers. While genetics knowledge was modestly improved, there is evidence of a negative effect of PGT on genetic selfefficacy, which may reflect an appropriate reevaluation of selfefficacy following receipt of complex genetic risk information. Regardless of the reason for the observed decrease in genetics self-efficacy, the association between genetics self-efficacy and each of decision regret, perceived control over health, and perceived value of PGT suggests that steps to promote genetic selfefficacy could positively impact customer satisfaction with PGT.

\section{ACKNOWLEDGMENTS}

The authors acknowledge the helpful comments of an anonymous reviewer regarding future directions for research in this area. The PGen Study is supported by the National Institutes of Health $(\mathrm{NIH})$ National Human Genome Research Institute (R01-HG005092). D.A.C. is supported by the Canadian Institutes of Health Research (Doctoral Foreign Study Award). K.A.K. is supported by the National Cancer Institute (R01-CA168608). R.C.G. also is supported by $\mathrm{NIH}$ grants U01-HG006500, U19-HD077671, and R01-HG002213. The content is solely the responsibility of the authors and does not necessarily represent the official views of the National Center for Research Resources, the National Institutes of Health, the National Cancer Institute, or the Canadian Institutes of Health Research. Members of the PGen Study at the time of publication are as follows: Robert C. Green, Joel B. Krier, Caroline M. Weipert, Sarah S. Kalia, Kurt D. Christensen, and Lisa S. Lehmann, Harvard Medical School and Brigham and Women's Hospital; Deanna Alexis Carere and Peter Kraft, Harvard School of Public Health; J. Scott Roberts, Mack T. Ruffin IV, Lan Q. Le, and Jenny Ostergren, University of Michigan School of Public Health; Wendy R. Uhlmann and Mick P. Couper, University of Michigan; Joanna L. Mountain and Amy K. Kiefer, 23andMe; Tanya A. Moreno and K. David Becker, Pathway Genomics; Scott D. Crawford, Survey Sciences Group; L. Adrienne Cupples, Clara A. Chen, and Catharine Wang, Boston University; Stacy W. Gray, Dana-Farber Cancer Institute; Barbara A. Koenig, University of California San Francisco; Kimberly Kaphingst, University of Utah; and Sarah Gollust, University of Minnesota.

\section{DISCLOSURE}

The authors declare no conflict of interest.

\section{REFERENCES}

1. Institute of Medicine of the National Academies. Health Literacy: a Prescription to End Confusion. The National Academies Press: Washington, DC, 2004.

2. Kutner M, Greenberg E, Jin Y, Paulsen C. The Health Literacy of America's Adults: Results From the 2003 National Assessment of Adult Literacy. US Department of Education, National Center for Education Statistics: Washington, DC, 2006.

3. Berkman ND, Sheridan SL, Donahue KE, Halpern DJ, Crotty K. Low health literacy and health outcomes: an updated systematic review. Ann Intern Med 2011;155:97-107.

4. Mitchell SE, Sadikova E, Jack BW, Paasche-Orlow MK. Health literacy and 30-day postdischarge hospital utilization. J Health Commun 2012;17 Suppl 3:325-338.

5. Lee JY, Divaris K, DeWalt DA, et al. Caregivers' health literacy and gaps in children's Medicaid enrollment: findings from the Carolina Oral Health Literacy Study. PLoS One 2014;9:e110178.

6. Osborn CY, Cavanaugh K, Wallston $\mathrm{KA}$, et al. Health literacy explains racial disparities in diabetes medication adherence. J Health Commun 2011;16 Suppl 3:268-278.

7. Omachi TA, Sarkar U, Yelin EH, Blanc PD, Katz PP. Lower health literacy is associated with poorer health status and outcomes in chronic obstructive pulmonary disease. J Gen Intern Med 2013;28:74-81.

8. Hurle B, Citrin T, Jenkins JF, et al. What does it mean to be genomically literate?: National Human Genome Research Institute Meeting Report. Genet Med 2013;15:658-663.

9. Lanie AD, Jayaratne TE, Sheldon JP, et al. Exploring the public understanding of basic genetic concepts. J Genet Couns 2004;13:305-320.

10. Ashida S, Goodman M, Pandya C, et al. Age differences in genetic knowledge, health literacy and causal beliefs for health conditions. Public Health Genomics 2011;14:307-316

11. Erblich J, Brown K, Kim Y, Valdimarsdottir HB, Livingston BE, Bovbjerg DH. Development and validation of a Breast Cancer Genetic Counseling Knowledge Questionnaire. Patient Educ Couns 2005;56:182-191.

12. Matloff ET, Moyer A, Shannon KM, Niendorf KB, Col NF. Healthy women with a family history of breast cancer: impact of a tailored genetic counseling intervention on risk perception, knowledge, and menopausal therapy decision making. J Womens Health (Larchmt) 2006;15:843-856.

13. Lerman C, Biesecker B, Benkendorf JL, et al. Controlled trial of pretest education approaches to enhance informed decision-making for BRCA1 gene testing. J Natl Cancer Inst 1997:89:148-157. 


\section{ORIGINAL RESEARCH ARTICLE}

14. Smerecnik CM, Mesters I, Verweij E, de Vries NK, de Vries H. A systematic review of the impact of genetic counseling on risk perception accuracy. J Genet Couns 2009;18:217-228.

15. Berkenstadt M, Shiloh S, Barkai G, Katznelson MB, Goldman B. Perceived personal control (PPC): a new concept in measuring outcome of genetic counseling. Am J Med Genet 1999;82:53-59.

16. Frueh FW, Greely HT, Green RC, Hogarth S, Siegel S. The future of directto-consumer clinical genetic tests. Nat Rev Genet 2011;12:511-515.

17. Evans JP, Green RC. Direct to consumer genetic testing: Avoiding a culture war Genet Med 2009;11:568-569.

18. Kaphingst KA, McBride CM, Wade C, et al. Patients' understanding of and responses to multiplex genetic susceptibility test results. Genet Med 2012;14:681-687.

19. Lehmann LS, Kaufman DJ, Sharp RR, et al. Navigating a research partnership between academia and industry to assess the impact of personalized genetic testing. Genet Med 2012;14:268-273.

20. Carere DA, Couper MP, Crawford SD, et al.; PGen Study Group. Design, methods, and participant characteristics of the Impact of Personal Genomics (PGen) Study, a prospective cohort study of direct-to-consumer personal genomic testing customers. Genome Med 2014;6:96.

21. 23andMe Inc. https://www.23andme.com/. Accessed 15 November 2014.

22. Pathway Genomics Corp. http://www.pathway.com. Accessed 15 November 2014

23. The White House Office of Management and Budget. Revisions to the standards for the classification of federal data on race and ethnicity. Executive Office of the President OoMaBO, Office of Information and Regulatory Affairs. The White House: Washington, DC, 1997.

24. Ware JE Jr, Gandek B. Overview of the SF-36 Health Survey and the International Quality of Life Assessment (IQOLA) Project. J Clin Epidemiol 1998:51:903-912.

25. Spitzer RL, Kroenke K, Williams JB, Löwe B. A brief measure for assessing generalized anxiety disorder: the GAD-7. Arch Intern Med 2006;166: 1092-1097.

26. Bowling BV, Acra EE, Wang $L$, et al. Development and evaluation of a genetics literacy assessment instrument for undergraduates. Genetics 2008;178:15-22.
27. Furr LA, Kelly SE. The Genetic Knowledge Index: developing a standard measure of genetic knowledge. Genet Test 1999;3:193-199.

28. Erby LH, Roter D, Larson S, Cho J. The rapid estimate of adult literacy in genetics (REAL-G): a means to assess literacy deficits in the context of genetics. Am J Med Genet A 2008;146A:174-181.

29. Molster C, Charles T, Samanek A, O'Leary P. Australian study on public knowledge of human genetics and health. Public Health Genomics 2009:12:84-91.

30. Smerecnik CM, Mesters I, de Vries NK, de Vries H. Alerting the general population to genetic risks: the value of health messages communicating the existence of genetic risk factors for public health promotion. Health Psychol 2009:28:734-745

31. Kaphingst KA, McBride CM, Wade C, Alford SH, Brody LC, Baxevanis AD. Consumers' use of web-based information and their decisions about multiplex genetic susceptibility testing. J Med Internet Res 2010;12:e41.

32. Parrott R, Silk K, Raup Krieger J, Harris T, Condit C. Behavioral health outcomes associated with religious faith and media exposure about human genetics. Health Commun 2004:16:29-45.

33. Brehaut JC, O'Connor AM, Wood TJ, et al. Validation of a decision regret scale. Med Decis Making 2003:23:281-292.

34. Bandura A. Social Foundations of Thought and Action: a Social Cognitive Theory. Prentice Hall: Englewood Cliffs, NJ, 1986.

35. Lachance CR, Erby LA, Ford BM, Allen VC Jr, Kaphingst KA. Informational content, literacy demands, and usability of websites offering health-related genetic tests directly to consumers. Genet Med 2010;12:304-312.

36. American Board of Genetic Counseling. Genetic counselors' scope of practice. http://www.abgc.net/docs/GC_Scope_of_prractice_final.pdf. Accessed 15 November 2014.

37. Veach PM, Bartels DM, Leroy BS. Coming full circle: a reciprocal-engagement model of genetic counseling practice. J Genet Couns 2007:16:713-728.

38. Samuel GN, Jordens CF, Kerridge I. Direct-to-consumer personal genome testing: ethical and regulatory issues that arise from wanting to 'know' your DNA. Intern Med J 2010;40:220-224.

39. Strecher VJ, DeVellis BM, Becker MH, Rosenstock IM. The role of self-efficacy in achieving health behavior change. Health Educ Q 1986;13:73-92. 